\title{
Adaptive Frequency Oscillators and Applications
}

\author{
Ludovic Righetti, ${ }^{*}$, Jonas Buchli $^{2}$ and Auke Jan Ijspeert ${ }^{1}$
}

${ }^{7}$ School of Communication and Computer Science, Ecole Polytechnique Fédérale de Lausanne, Lausanne, CH-1015 Switzerland

${ }^{2}$ Computational Learning and Motor Control Lab, University of Southern California, Los Angeles, CA 90089, USA

\begin{abstract}
In this contribution we present a generic mechanism to transform an oscillator into an adaptive frequency oscillator, which can then dynamically adapt its parameters to learn the frequency of any periodic driving signal. Adaptation is done in a dynamic way: it is part of the dynamical system and not an offline process. This mechanism goes beyond entrainment since it works for any initial frequencies and the learned frequency stays encoded in the system even if the driving signal disappears. Interestingly, this mechanism can easily be applied to a large class of oscillators from harmonic oscillators to relaxation types and strange attractors. Several practical applications of this mechanism are then presented, ranging from adaptive control of compliant robots to frequency analysis of signals and construction of limit cycles of arbitrary shape.
\end{abstract}

\section{INTRODUCTION}

Nonlinear oscillators are very important modeling tools in biological and physical sciences, and these models have received particular attention in many engineering fields over the last few decades. The models are interesting because of their capability to synchronize with other oscillators or with external driving signals. However, these synchronization capabilities are limited, and it is not always an easy task to correctly choose the model parameters to ensure proper synchronization with the external driving signals. Indeed, an oscillator has a finite entrainment region which depends on many parameters, such as the coupling strength and the frequency difference between the oscillator and the driving signal.

Recent work, however, has shown that it is possible to modify nonlinear oscillators so that they can overcome the limitations above, by adding dynamics to the parameters of an individual oscillator, allowing it to learn the frequency of an input signal. These attempts are often limited to simple classes of oscillators, equivalent to phase oscillators $[1,2]$ or to simple classes of driving signal (pulses) [3].

Recently we designed a learning mechanism for oscillators, which adapts the oscillator frequency to the frequency of any periodic input signal $[4,5]$. The parameter with the strongest influence on the frequency of the oscillator is turned into a new state variable for the system. Interestingly, this mechanism appears to be generic enough to be applied to many different types of oscillators, from phase oscillators to relaxation types, and to strange attractors. The frequency adaptation process goes beyond mere entrainment, because, even if the input signal disappears, the learned frequency stays encoded in the

*Address correspondence to this author at the School of Communication and Computer Science, Ecole Polytechnique Fédérale de Lausanne, Lausanne, CH-1015 Switzerland; E-mail: ludovic.righetti@a3.epfl.ch oscillator. Moreover, it is independent of the initial conditions, thus working beyond entrainment basins (i.e. it has an infinite basin of attraction). We call this adaptation mechanism dynamic Hebbian learning because it shares similarities with correlationbased learning observed in neural networks [6].

In this contribution, we present our generic adaptation mechanism. Then we demonstrate several applications, ranging from adaptive control of legged robots with passive dynamics [4, 7], where the adaptive oscillators find the resonant frequency of the robot, to frequency analysis with systems of coupled adaptive oscillators [8], and finally to construction of limit cycles with arbitrary shape [9].

\section{ADAPTIVE FREQUENCY OSCILLATORS}

\section{A. A generic Rule for Frequency Adaptation}

We consider general equations for an oscillator perturbed by a periodic driving signal

$$
\begin{aligned}
& \dot{x}=f_{x}(x, y, \omega)+K F(t) \\
& \dot{y}=f_{y}(x, y, \omega)
\end{aligned}
$$

where $f_{x}$ and $f_{y}$ are functions of the state variables that produce a structurally stable limit cycle, and of a parameter $\omega$ that has a monotonic relation with the frequency of the oscillator when unperturbed, $K=0$ (we do not require this relation to be linear). $F(t)$ is a time periodic perturbation and $\mathrm{K}>0$ the coupling strength.

In order to enable the oscillator to learn the frequency of $F(t)$, we transform the $\omega$ parameter into a new state variable, with its own dynamics. The generic rule that allows us to transform the basic oscillator into an adaptive frequency oscillator is as follows:

$$
\dot{\omega}= \pm K F(t) \frac{y}{\sqrt{x^{2}+y^{2}}}
$$


where the sign depends on the direction of rotation of the limit cycle in the $(\mathrm{x}, \mathrm{y})$ plane.

\section{B. Properties of the Adaptation Mechanism}

We proved in [5] that the adaptation mechanism causes an oscillator's frequency to converge to the frequency of any periodic input signal, for phase and Hopf oscillators. In the case where there are several frequencies in the spectrum of $F(t)$, the oscillator converges to one input frequency component, depending on the initial frequency of the oscillator.

Further, the higher the coupling strength $\mathrm{K}$, the faster convergence occurs. It can be shown that for suitable coupling strength, the convergence is exponential (of order $e^{-t}$ ) [10]. Examples of frequency adaptation for the Hopf
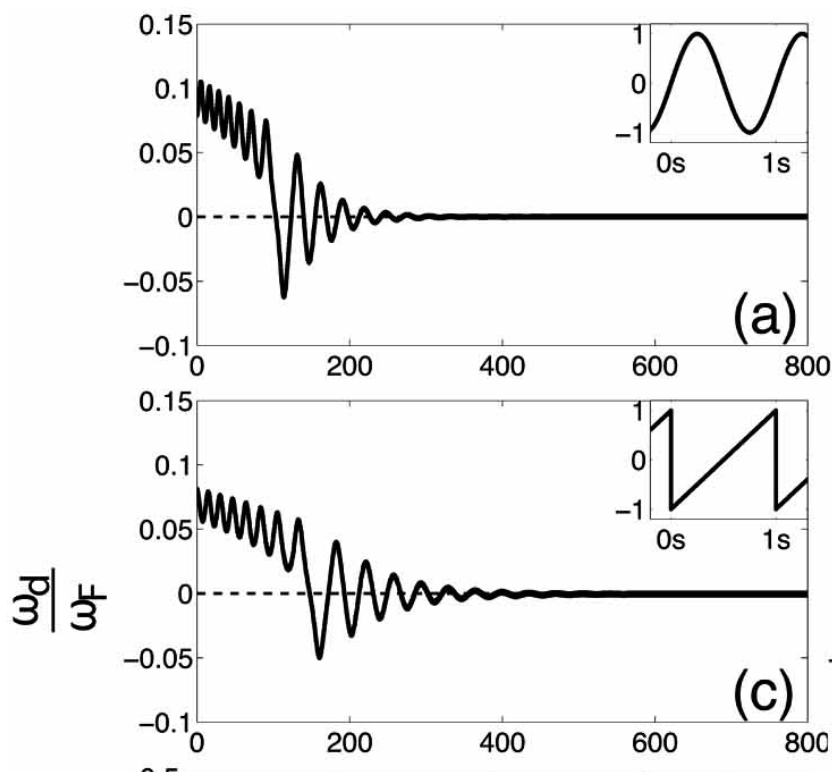

(a)
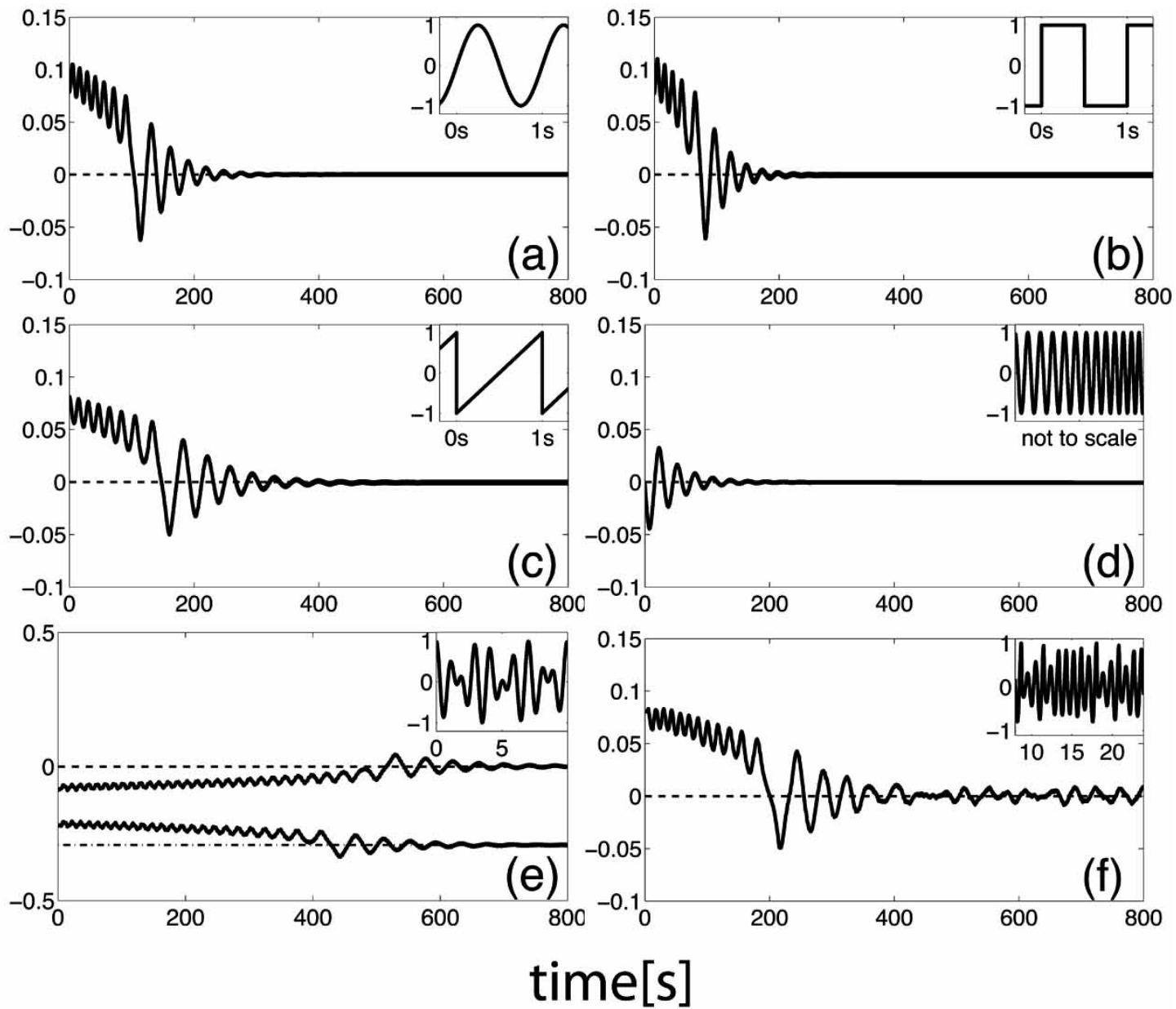

Fig. (1). (a) Typical convergence of an adaptive frequency Hopf oscillator driven by a harmonic signal $((\mathrm{F}(\mathrm{t})=\sin (2 \pi \mathrm{t}))$. The frequencies converge towards the frequency of the input (indicated in dashed line). After convergence the frequency oscillates with a small amplitude around the frequency of the input. In all figures, we plot in the main graph the time evolution of the difference between $\omega$ and the input frequency, normalized by the input frequency. The top right panel shows the driving signals (note the different scales). (b) Square pulse $\mathrm{F}(\mathrm{t})=\operatorname{rect}\left(\omega_{\mathrm{F}} \mathrm{t}\right),(\mathbf{c})$ Sawtooth, $\mathrm{F}(\mathrm{t})=\operatorname{st}\left(\omega_{\mathrm{F}} \mathrm{t}\right),(\mathbf{d})$ Chirp $\mathrm{F}(\mathrm{t})=\cos \left(\omega_{\mathrm{c}} \mathrm{t}\right)$, where $\omega_{\mathrm{c}}=\omega_{\mathrm{F}}\left(1+\frac{1}{2}\left(\frac{\mathrm{t}}{1000}\right)^{2}\right)$. (Note that the graph of the input signal is illustrative only since changes in frequency takes much longer than illustrated). (e) Signal with two non-commensurate frequencies $\mathrm{F}(\mathrm{t})=\frac{1}{2}\left[\cos \left(\omega_{\mathrm{F}} \mathrm{t}\right)+\cos \left(\frac{\sqrt{2}}{2} \omega_{\mathrm{F}} \mathrm{t}\right)\right]$, i.e. a representative example how the system can evolve to different frequency components of the driving signal depending on the initial condition $\omega_{d}(0)=\omega(0)-\omega_{\mathrm{F}}$. (f) $F(t)$ is the non-periodic output of the Rössler system. The Rössler signal has a 1/f broad-band spectrum, yet it has a clear maximum in the frequency spectrum. In order to assess the convergence we use $\omega_{\mathrm{F}}=2 \pi f_{\max }$, where $f_{\max }$ is found numerically by FFT. The oscillator converges to this frequency. 


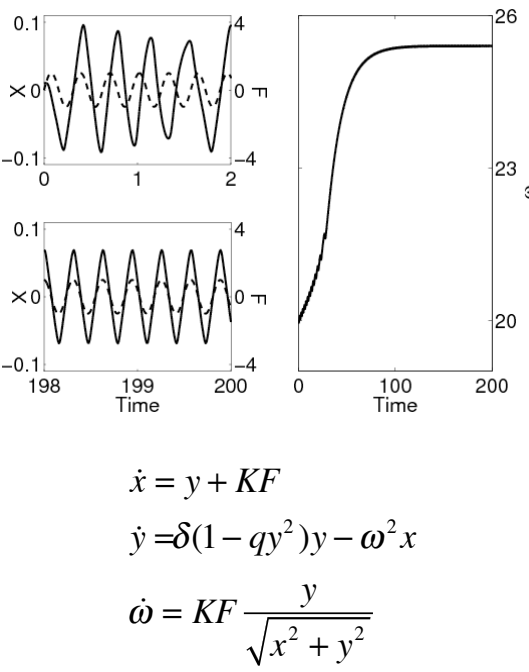

(a) Adaptive Rayleigh oscillator

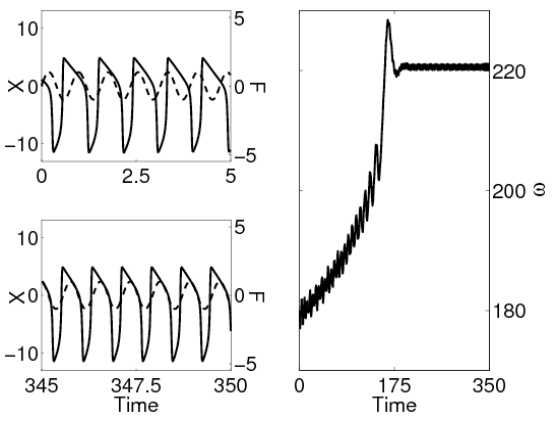

$$
\begin{aligned}
& \dot{x}=x(x-a)(1-x)-y+K F \\
& \dot{y}=\omega(x-b y) \\
& \dot{\omega}=-K F \frac{y}{\sqrt{x^{2}+y^{2}}}
\end{aligned}
$$

(b) Adaptive Fitzhugh-Nagumo oscillator

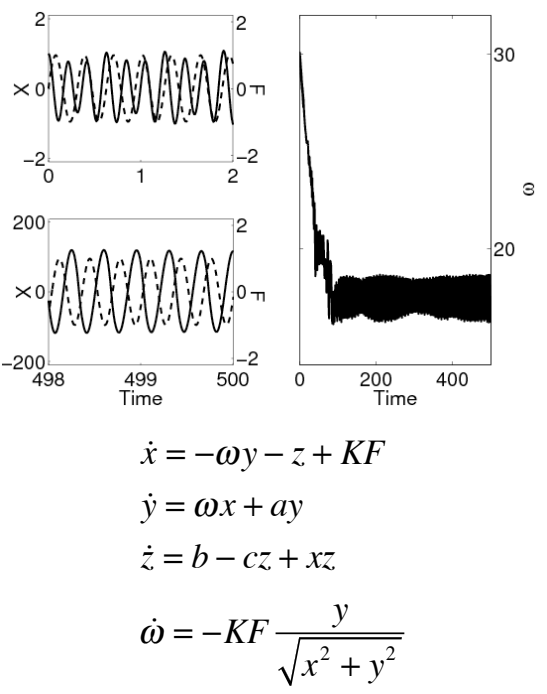

(c) Adaptive Rössler system

Fig. (2). For each oscillator, $\omega$ corresponds to the adaptive parameter. Each figure is composed of 3 plots. The right plot shows the evolution of $\omega$. The left plots are the time evolution of the oscillators (the x variable) and of the input signal $F$ (dashed line), before (upper plot) and after (lower plot) adaptation.

Our extensive numerical simulations also show that this adaptation mechanism works for many different types of non-harmonic oscillators. Some examples, shown in Fig. (2), are an adaptive Rayleigh oscillator, an adaptive FitzhughNagumo oscillator and a Rössler system in chaotic mode. For the first two cases there is no linear relation between $\omega$ and the frequency of oscillations, but the adaptive mechanism is able to find a suitable value for $\omega$ such that the frequency of the oscillator is the same as the frequency of the input signal. For the Rössler system, the frequency of the system is not well defined since the system is not periodic, but we can define a pseudo-frequency and the system can then adapt it to the frequency of a periodic input.

\section{APPLICATIONS}

We now present several applications for the adaptation mechanism, ranging from robot control to frequency analysis and automatic construction of limit cycles of arbitrary shape.

\section{A. Robot with Passive Dynamics}

The adaptation mechanism can be used to find the resonant frequencies of legged robots with passive elements (i.e. springs) $[4,7,11,12]$. A controller based on adaptive frequency oscillators is able to tune itself to the resonant frequency of the robot, via a simple feedback loop using sensors on-board (e.g. position or inertial sensors). Locomotion can therefore be made very efficient by exploiting the intrinsic dynamics of the robot. Another advantage is that one does not need to tune the controller for a specific robot; the controller can also track any changes in resonant frequency automatically, if, for example, the frequency changes due to a variation in mass or spring stiffness, or because of a gait transition (e.g. the resonant frequency is different if the robot is standing on two feet or four feet).

\section{B. Frequency Analysis}

Another application is the use of a pool of adaptive frequency Hopf oscillators to perform frequency analysis on an input signal [8]. The oscillators are coupled via a negative mean field with the input teaching signal, as is shown in Fig. (3). The oscillators converge to the frequencies present in the spectrum of the teaching signal and due to the negative feedback, each time an oscillator finds a correct frequency, this one loses its amplitude. Thus, the other oscillators only feel the remaining frequencies to learn.

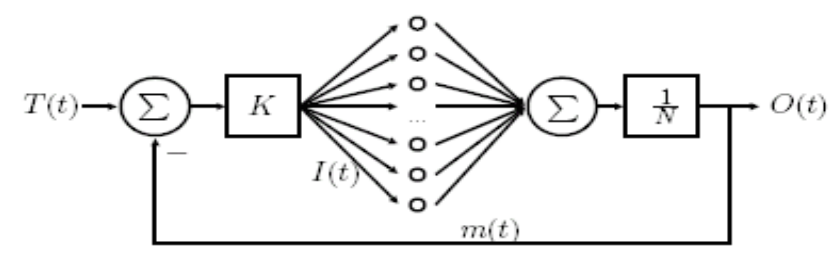

Fig. (3). Structure of the pool of adaptive frequency oscillators that is able to reproduce a given teaching signal $\mathrm{T}(\mathrm{t})$. The mean field produced by the oscillators is fed back negatively to the oscillators.

The pool of oscillators is able to approximate the frequency spectrum of any input signal. This works for signals with discrete spectra, and also for those with continuous and time-varying spectra. The spectrum is approximated by the distribution of the frequencies of the oscillators, and so the resolution of the approximation can be made arbitrary good by increasing the number of oscillators in the pool.

Fig. (4) shows how the system can approximate the spectrum of a broad-band chaotic signal from the Rössler system. As can be seen, the important features of the 


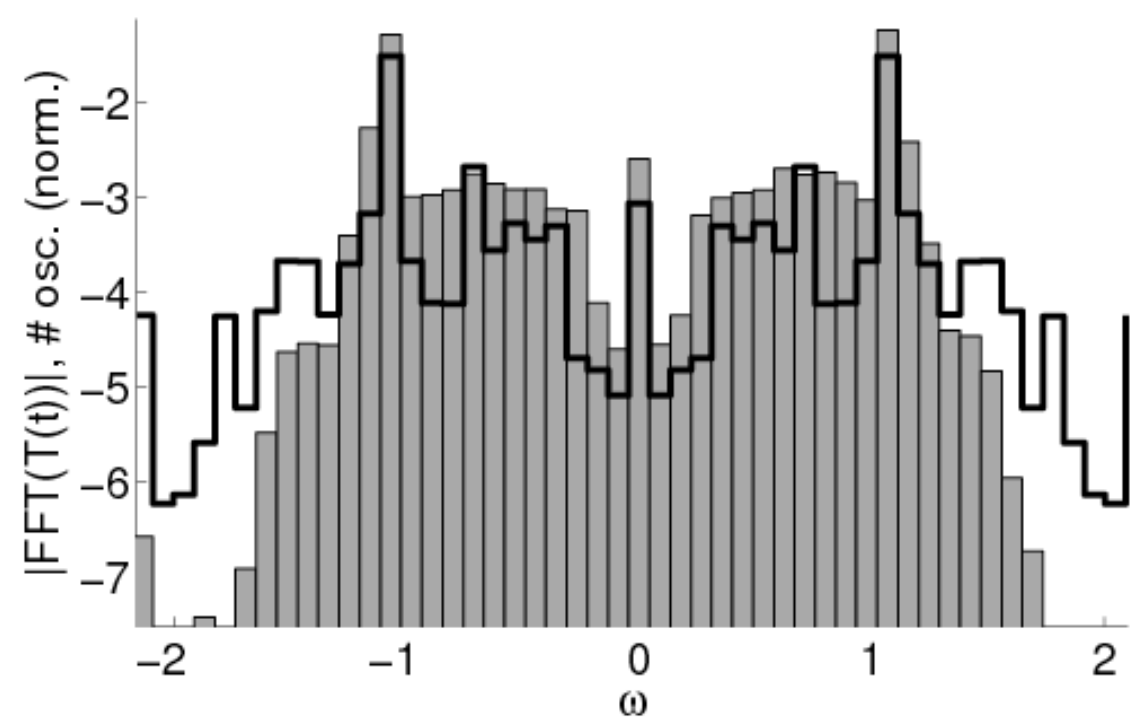

Fig. (4). FFT of the Rössler signal (black line) in comparison with the distribution of the frequencies of the oscillators (gray bars normalized to the number of oscillators, $\mathrm{N}=10000$ ). The spectrum of the signal has been discretized into the same bins as the statistics of the oscillators in order to allow for comparison with the results from the full-scale simulation.

spectrum are caught by the system, especially the broad spectrum and the major frequency peaks.

\section{Construction of Limit Cycles with Arbitrary Shape}

The previous pool of oscillators can be extended by adding a weight to each oscillator in the mean field sum, and a coupling between oscillators, in order to ensure stability of the output pattern. The result is that an individual oscillator will be able to fully match the energy content of a frequency in the spectrum of the teaching signal. Moreover, the coupling ensures that the system exhibits a stable limit cycle. Here, amplitudes and phase differences become system state variables, in addition to frequencies. The governing differential equations of the system are then:

$\dot{x}_{i}=\left(\mu-r_{i}^{2}\right) x_{i}-\omega_{i} y_{i}+K F(t)+\tau \sin \left(\frac{\omega_{i}}{\omega_{0}} \theta_{0}-\theta_{i}-\phi_{i}\right)$

$\dot{y}_{i}=\left(\mu-r_{i}^{2}\right) y_{i}+\omega_{i} x_{i}$

$\dot{\omega}_{i}=-K F(t) \frac{y_{i}}{r_{i}}$

$\dot{\alpha}_{i}=\eta x_{i} F(t)$

$\dot{\phi}_{i}=\sin \left(\frac{\omega_{i}}{\omega_{0}} \theta_{0}-\theta_{i}-\phi_{i}\right)$

with

$r_{i}^{2}=x_{i}^{2}+y_{i}^{2}$

$\theta_{i}=\operatorname{sgn}\left(x_{i}\right) \cos ^{-1}\left(-\frac{y_{i}}{r_{i}}\right)$

$F(t)=P_{\text {teach }}(t)-Q_{\text {learned }}(t)$

$Q_{\text {learned }}(t)=\sum_{i=0}^{N} \alpha_{i} x_{i}$ where $\tau, K$ and $\eta$ are positive constants. The output of the system, $Q_{\text {learned }}$, is the weighted sum of the output of each oscillator. $F(t)$ represents the negative feedback, which on average is the remainder of the teaching signal that $P_{\text {teach }}(t)$ the network still has to learn. $\alpha_{i}$ represents the amplitude associated with the frequency $\omega_{i}$ of oscillator $i$. The evolution equation maximizes the correlation between $x_{i}$ and $F(t)$, which means that $\alpha_{i}$ will increase only if $\omega_{i}$ has converged to a frequency component of $F(t)$ (the correlation will be positive on average) and will stop increasing when the frequency component $\omega_{i}$ disappears from $F(t)$ because of the negative feedback loop. $\phi_{i}$ is the phase difference between oscillator $i$ and 0 . The value converges to the phase difference between the instantaneous phase of oscillator $0, \theta_{0}$, scaled for frequency $\omega_{i}$, and the instantaneous phase of oscillator $i, \theta_{i}$. Each adaptive oscillator is coupled with oscillator 0 , with strength $\tau$, to maintain the correct phase relationships between oscillators.

Fig. (5) shows an example of the convergence of a network of oscillators with amplitudes and coupling, together with the resulting learned signal. We see that the individual oscillator frequencies first converge to the frequency components present in the teaching signal. Individual amplitudes increase when the associated frequency matches one frequency of the input signal. Finally, the phase differences stabilize and we see that the error is zero, which means that the system has perfectly reconstructed the teaching signal. Further, the teaching signal is now encoded into a structurally stable limit cycle and it is easy to smoothly modulate its frequency and amplitude by changing $\vec{\omega}$ and $\vec{\alpha}$. These properties can be very useful, together with sensory feedback, for robotics control (see for example [9]). This system can be viewed as a dynamic Fourier series decomposition where there is no need of explicitly define a time window or to perform any preprocessing of the input signal. 

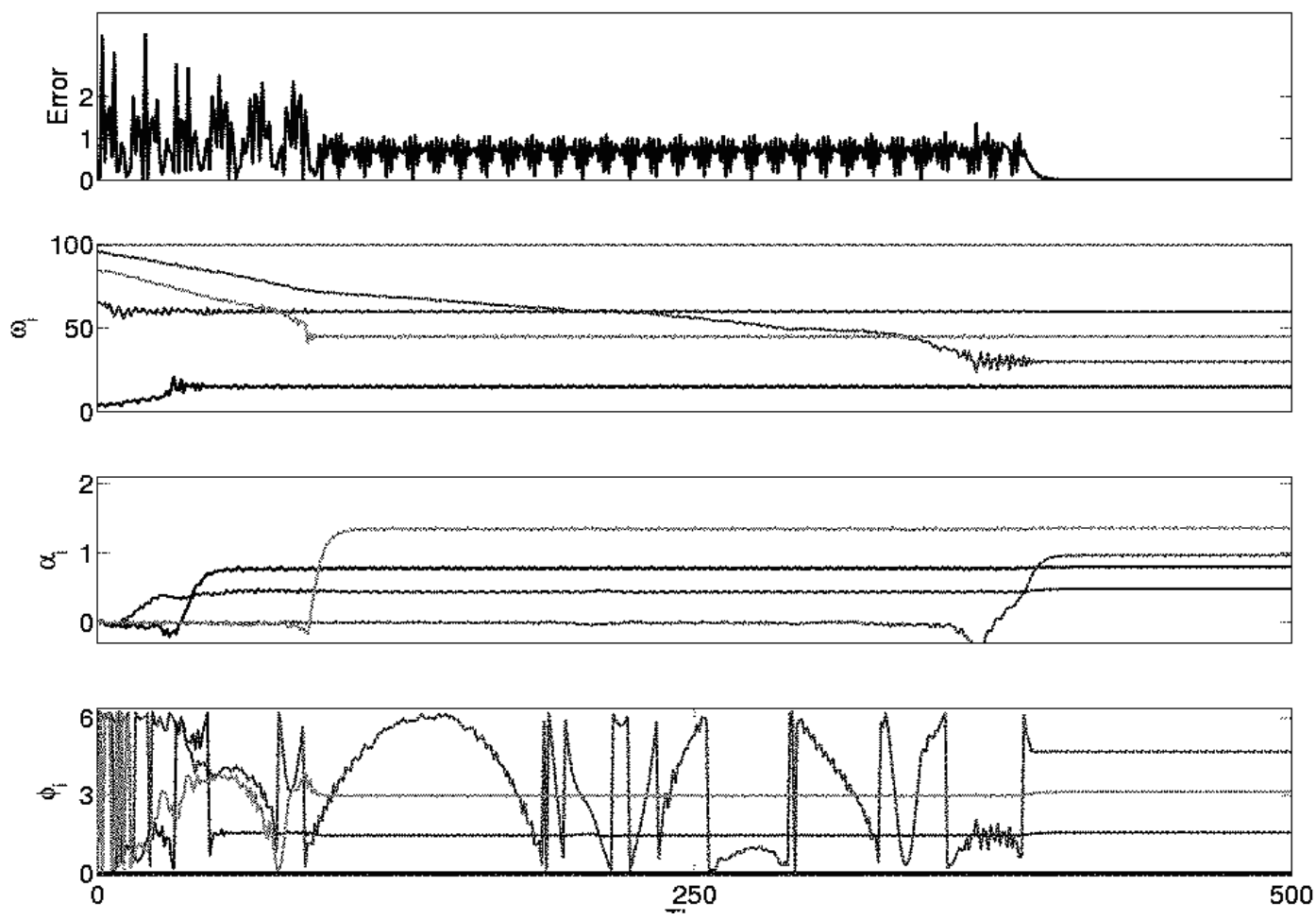

(a) Evolution of the state variables of the system
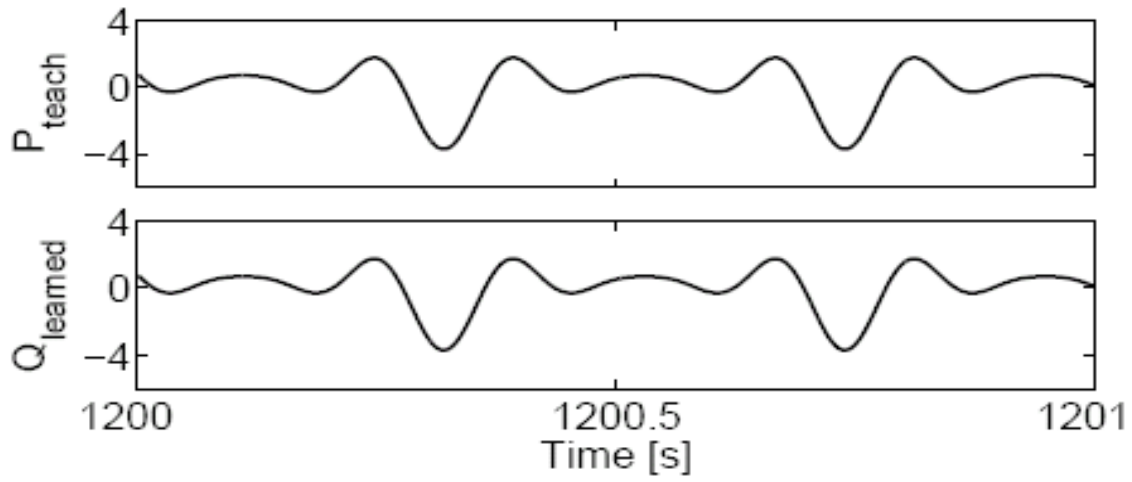

(b) Result of learning

Fig. (5). Construction of a limit cycle by learning an input signal $\left(P_{\text {teach }}=0.8 \sin (15 t)+\cos (30 t)-1.4 \sin (45 t)-0.5 \cos (60 t)\right)$. Fig. (5a) shows the evolution of the state variables of the system during learning. The upper graph is a plot of the error $\left(\left\|P_{\text {teach }}-Q_{\text {learned }}\right\|\right)$. The 3 other graphs show the evolution of the frequencies, $\omega_{i}$, the amplitudes, $\alpha_{i}$ and the phases, $\phi_{i}$. We clearly see that the system can learn the teaching signal perfectly - the frequencies, amplitudes and phase differences converge to the correct values and the error becomes zero. Fig. (5b) shows the result of learning (teaching signal in upper graph, output of the system in lower graph), we note the perfect reconstruction of the signal.

\section{CONCLUSION}

In this contribution we presented a generic mechanism for building adaptive frequency oscillators from a given, existing oscillator. We showed that our approach can be applied to many different types of oscillators, and that the resulting systems are able to learn the frequencies of any periodic input signal. Interestingly, there is no need to preprocess the signal and no external optimization procedures are required to obtain the correct frequency. All the learning is embedded in the dynamics of the adaptive oscillators. Moreover, our results go further than entrainment, since the learned frequency is maintained in the system even if the external driving oscillation disappears and the basin of attraction is infinite (i.e. the system can start from any initial frequency). Finally, we discussed some applications of this mechanism, ranging from adaptive control for compliant robots, to frequency analysis and construction of limit cycles of arbitrary shape. 


\section{ACKNOWLEDGEMENT}

This work was supported by the European Commission's Cognition Unit, project no. IST-2004-004370: RobotCub (L.R.) and by a grant from the Swiss National Science Foundation (L.R, J.B. and A.I.).

\section{REFERENCES}

[1] R. Borisyuk, M. Denham, F. Hoppensteadt, Y. Kazanovich, and O. Vinogradova, "Oscillatory model of novelty detection", Netw. Comput. Neural Syst., vol. 12, pp. 1-20, 2001.

[2] J. Nishii, "A learning model for oscillatory networks," Neural Netw., vol. 11, pp. 249-257, 1998.

[3] E. Large and J. Kolen, "Resonance and the perception of musical meter," Connect. Sci., vol. 6, pp. 177-208, 1994.

[4] J. Buchli and A. Ijspeert, "A simple, adaptive locomotion toysystem," In: From Animals to Animats 8., Proceedings of the Eighth International Conference on the Simulation of Adaptive Behavior (SAB'04), S. Schaal, A. Ijspeert, A. Billard, S. Vijayakumar, J. Hallam, and J. Meyer, Eds. MIT Press, 2004, pp. 153-162.

[5] L. Righetti, J. Buchli, and A. Ijspeert, "Dynamic hebbian learning in adaptive frequency oscillators," Physica. D, vol. 216, no. 2, pp. 269-281, 2006. R. Kempter, W. Gerstner, and J. van Hemmen, "Hebbian learnin
and spiking neurons," Phys. Rev. E, vol. 59, pp. 4498-4514, 1999. J. Buchli, F. Iida, and A. Ijspeert, "Finding resonance: Adaptive frequency oscillators for dynamic legged locomotion," In: Proceedings of the IEEE/RSJ International Conference on Intelligent Robots and Systems (IROS), 2006, pp. 3903-3909.

[8] J. Buchli, L. Righetti, and A. Ijspeert, "Frequency analysis with coupled nonlinear oscillators," Physica. D, vol. 237, no. 13, pp. $1705-1718,2008$.

[9] L. Righetti, and A. Ijspeert, "Programmable central pattern generators: an application to biped locomotion control," In: Proceedings of the 2006 IEEE International Conference on Robotics and Automation, Conference, May 2006, pp. 1585-1590,

[10] L. Righetti, "Control of locomotion using dynamical systems: design methods and adaptive frequency oscillators," Ph.D. dissertation, Ecole Polytechnique Fédérale de Lausanne, November 2008

[11] J. Buchli, L. Righetti, and A. Ijspeert, "A dynamical systems approach to learning: a frequency-adaptive hopper robot," In: Proceedings of the VIIIth European Conference on Artificial Life ECAL 2005, ser. Lecture Notes in Artificial Intelligence. Springer Verlag, Conference, 2005, pp. 210-220.

[12] J. Buchli, and A. Ijspeert, "Self-organized adaptive legged locomotion in a compliant quadruped robot," Autonom. Robots, vol. 25 , no. 4 , pp. 331-347, 2008.

This is an open access article licensed under the terms of the Creative Commons Attribution Non-Commercial License (http://creativecommons.org/licenses/by$\mathrm{nc} / 3.0 /$ ) which permits unrestricted, non-commercial use, distribution and reproduction in any medium, provided the work is properly cited.

\section{ABOUT AUTHORS}

Ludovic Righetti is working towards a PhD at the Biologically Inspired Robotics Group (BIRG) at the Ecole
Polytechnique Féderale de Lausanne (EPFL). He has a BSc/MSc in Computer Science from EPFL (March 2004). He
is particularly interested in the theoretical aspects of locomotion, both in animals and legged robots, and more
generally in control theory and dynamical systems.

